\title{
Diseño ergonómico del puesto de trabajo de cajera en supermercado con exposición a posturas incómodas.
}

\begin{abstract}
(c) (1)(9)
\end{abstract}
Ergonomic design of the cashier job in supermarkets with exposure to uncomfortable postures.

Lilian Adriana Cevallos Tapia. ${ }^{1}$, Manolo Alexander Córdova Suárez. ${ }^{2}$, Vladimir Vega Falcón. ${ }^{3} \&$ Edison Patricio Villacres Cevallos. ${ }^{4}$

\begin{abstract}
.
Introduction. The use of risk significance analysis for ergonomic job design is an alternative that can help reduce harmful effects on the health of workers and pay fines from employers. Objetives. A workstation was ergonomically designed with exposure to uncomfortable postures for the cashier in supermarkets belonging to the La Favorita company located in Ambato (Mall de los Andes), for which the critical variables to be controlled with the Rapid Upper Limb Assessment (RULA) method were established and then designed the position anthropometrically to improve posture. Methodology. The design results determine the following angles: arms $20^{\circ}-45^{\circ}$; forearm: $60^{\circ}-100^{\circ}$; pronation wrist; neck: $0^{\circ}-20^{\circ}$ and legs with support. The final risk level of RULA on both sides of the body was lowered by $66.6 \%$ by changing the reception table $90^{\circ}$ from the front and the dispatch table on the right side. As conclusions of the study, we can say that the level of risk due to forced postures decreases 4 points from the final RULA result, considering the workstation design proposal, the wrist must exercise pronation, and the worker must stand with the weight of the body distributed in both legs, and on the work,

\footnotetext{
${ }^{1}$ Universidad Regional Autónoma de los Andes, Facultad de Ciencias Médicas, Ambato, Tungurahua, pg.lilianact74@uniandes.edu.ec, http://orcid.org/0000-0002-8530-2870

2 Universidad Nacional de Chimborazo, Facultad de Ingeniería, Riobamba-Ecuador, hesconsultores@ hotmail.com, http://orcid.org/0000-0001-6786-7926

${ }^{3}$ Universidad Regional Autónoma de los Andes, Facultad de Ciencias Médicas, Ambato, Tungurahua, ua.vladimirvega@uniandes.edu.ec, http://orcid.org/ 0000-0003-0140-4018

4 Universidad Nacional de Chimborazo, Facultad de Ingeniería, Riobamba-Ecuador, pvillacres@ unach.edu.ec, http://orcid.org/0000-0001-9518-1278
} 
surface must be rotated $90^{\circ}$ and the desk for dispatch on the right side. Conclution. The ergonomic redesign of the supermarket cashier's workplace decreased the RULA risk level of the upper extremities by $66.66 \%$.

Keywords: Ergonomic design, work postures, RULA.

\section{Resumen.}

Introducción. El uso de análisis de significación de riesgos para diseño ergonómico de puestos de trabajo es una alternativa que puede ayudar en la disminución de efectos nocivos en la salud de los trabajadores y en pagos de multas de los empleadores Objetivo. Se diseñó ergonómicamente un puesto de trabajo con exposición a posturas incómodas para la cajera en supermercados pertenecientes a la empresa La Favorita ubicada en Ambato (Mall de los Andes), para lo cual se establecieron las variables críticas a controlar con el método: Rapid Upper Limb Assessment (RULA) y luego se diseñó el puesto antropométricamente para mejorar la postura. Metodología. El estudio empezó con la identificación de los ángulos de las extremidades superiores y el cuerpo, así como sus penalizaciones por la ejecución de la actividad. Luego se realizó un estudio de comparación de las variables con el método RULA para identificar los elementos que son factibles de modificación. Para finalmente escoger una altura adecuada del plano de trabajo. Resultados. Los resultados del diseño determinan los siguientes ángulos: brazos $20^{\circ}-45^{\circ}$; antebrazo: $60^{\circ}-100^{\circ}$; muñeca con pronación; cuello: $0^{\circ}-20^{\circ}$ y piernas con apoyo. Se bajó un 66,6\% el nivel de riesgo final de RULA en los dos lados del cuerpo con cambiar la mesa de recepción $90^{\circ}$ de frente y la mesa para despacho en el lado derecho. Como conclusiones del estudio podemos decir que el nivel de riesgo por posturas forzadas disminuye 4 puntos del resultado final RULA considerando la propuesta de diseño de puesto de trabajo, la muñeca debe ejercer pronación además el trabajador debe estar de pie con el peso del cuerpo distribuido en ambas piernas, y sobre el plano de trabajo debe girarse $90^{\circ}$ y la mesa para despacho en el lado derecho. Las características del puesto son para la región sierra y condiciones normales de presión y temperatura. Conclusión. El rediseño ergonómico del puesto de trabajo de cajera de supermercado disminuyó el nivel de riesgo RULA de las extremidades superiores en un 66,66\%.

Palabras claves: Diseño ergonómico, posturas de Trabajo, RULA.

\section{Introducción.}

La ergonomía es una ciencia interdisciplinaria encargadas del estudio del equilibrio entre las condiciones externas e internas que se encuentran ligadas al trabajo, las mismas interaccionan en la biología humana con las exigencias y requerimientos de los sistemas y procesos de trabajo delimitando así su ámbito de aplicación para la preservación, corrección o mejora de medidas preventivas. (Quintana Salgado LA, 2020). Las condiciones de trabajo con exposición al riesgo por la adopción de posturas inadecuadas, 
genera fatiga y con el transcurso del tiempo puede causar problemas graves en la salud del trabajador. (Henao Robledo, 2016).

El manejo manual de cargas aunque menor en peso a $1 \mathrm{~kg}$, la adopción de posturas inadecuadas en el trabajo resulta algo frecuente, por lo cual, para llevar a cabo la evaluación de dicha actividad, la frecuencia con que se maneja la carga así como su peso y estabilidad del contenido en el interior de la carga podría dificultar su traslado y causar en la persona trastornos músculo esqueléticos en los colaboradores de los supermercados (Ortiz, 2018) que a medio o largo plazo, pueden llegar a provocar patologías laborales (Beatriz Capezzuto, 2017).

La evaluación de las denominadas posturas ocupadas en el trabajo debe ser en la sintaxis de ángulos y pueden realizarse directamente además si se ocupa fotografías para mayor facilidad estas deben ser de ángulos distintos para complementar la evaluación (Moreno Medina, 2018). Al evaluar dichas posturas, se debe hacer énfasis en las posturas individuales que toma el trabajador, con esto debe prestarse atención a los ángulos de flexión o semi flexión formados en las extremidades, así como el esfuerzo necesario empleado (Diego Mas, 2015). En este caso el método de evaluación RULA se implementa para el análisis individual de las posturas optadas por el trabajador, además este considera la repetitividad de los movimientos y la actividad estática del sistema musculoesquelético (Moya Reyes, 2017).

Las actividades desarrolladas en los supermercados son la mayor parte estáticas y los trabajadores se adaptan a los entornos laborales fomentando la aparición de muchos problemas y manifestaciones tempranas en su salud. Los nuevos escenarios de trabajo consideran la ergonomía como sistema fundamental para prevenir enfermedades y sobre todo evitar problemas legales y multas que a la larga causan ausentismo laboral (Pablo E. Aliaga, 2016).

El rediseño de las estaciones de trabajo del puesto de cajera resulta relevante para cuidar la salud de los trabajadores y evitar ausentismo por bajas inesperadas debido a una posible enfermedad, derivada de su puesto de trabajo. Este trabajo analiza las opciones de un $\}$ mejora que beneficie al trabajador y evite problemas al empleador por sanciones o enfermedades ocupacionales. (Levano Boggiano, 2018).

\section{Materiales y métodos}

Este trabajo siguió los siguientes pasos: a) Estudio de trabajo, b) Evaluación de las posturas, c) análisis de las variables, d) diseño ergonómico (Moreno Fiallos Victor, 2019).

\section{Estudio de trabajo}

Se realizó primero una selección de las actividades, tareas, tiempos de ejecución de los procesos relacionados con la adopción de posturas incómodas de las extremidades superiores, además se consideró datos propios del personal que ejecuta el trabajo 
(Avendaño Avendaño Sandra Bibiana, 2020) En esta etapa se identificó los datos de entrada del método RULA para los dos lados del cuerpo del trabajador. No fue necesario una muestra poblacional debido a que la ejecución de la tarea adopta los mismos ángulos a la mayoría de las personas que ocupan es puesto de trabajo (Kolodziej, 2019). La investigación se enfocó en la realidad de Ecuador, afrontando el problema mediante la aplicación del método científico de RULA (Gómez, 2017).

\section{Evaluación de las posturas de trabajo, método RULA}

Se aplica el método RULA para realizar el diseño del puesto de trabajo, este método es uno de los más utilizados para el análisis de extremidades superiores con resultados exitosos (Jara, 2018).

La aplicación del método necesita la medición de ángulos y trabajar en base a las puntuaciones presentadas en las tablas del método considerando que se hace por grupos pues a partir de estas se hará una puntuación final, dicho valor es proporcional al riesgo que conlleva realizar eta actividad. (Saúl Rangel Lara, 2019). Si la puntuación es muy alta significa que la actividad es muy riesgosa y que implica la aparición de lesiones a largo plazo, por lo que será necesaria la aplicación de medidas correctivas que deberán aplicarse en un lapso determinado de tiempo según su gravedad. (Campos, 2017).

Se empieza determinado los valores del grupo A empezando con los brazos ver tabla 1.

Tabla 1. Puntuación brazos

\begin{tabular}{ll}
\hline Posición & Puntuación \\
\hline Desde $20^{\circ}$ de extensión a $20^{\circ}$ de flexión & $\mathbf{1}$ \\
Extensión $>20^{\circ}$ o flexión $>20^{\circ}$ y $<45^{\circ}$ & $\mathbf{2}$ \\
Flexión $>45^{\circ}$ y $90^{\circ}$ & $\mathbf{3}$ \\
Flexión $>90^{\circ}$ & $\mathbf{4}$ \\
\hline
\end{tabular}

Nota: La medición de brazos se obtiene del grado flexión/ extensión. Si el hombro esta elevado, brazos abducidos de suma 1 punto, si existe algún apoyo se resta 1 punto. Fuente: (Cuellar Mendoza Jonathan, 2018).

Se escoge la posición de los antebrazos, ver tabla 2.

Tabla 2. Puntuación antebrazo

\begin{tabular}{ll}
\hline Posición & Puntuación \\
\hline Flexión entre $60^{\circ}$ y $100^{\circ}$ & $\mathbf{1}$ \\
Flexión $\angle 60^{\circ}$ o $>100^{\circ}$ & $\mathbf{2}$
\end{tabular}

Nota: La medición del antebrazo se obtiene del ángulo de flexión. Si los antebrazos están a un lado del cuerpo o cruzan la línea media se suma 1 punto. Fuente: (Flores, 2018).

Se determina la puntuación de la muñeca con la tabla 3. 
Tabla 3. Puntuación muñeca

\begin{tabular}{ll}
\hline Posición & Puntuación \\
\hline Posición neutra & $\mathbf{1}$ \\
Flexión o extensión $>00^{\circ}$ y $<15^{\circ}$ & $\mathbf{2}$ \\
Flexión o extensión $>15^{\circ}$ & $\mathbf{3}$ \\
\hline
\end{tabular}

Nota: La medición de la muñeca se obtiene del ángulo de flexión/ extensión. Fuente: (Jessica Jazmin Pesina Lumbreras, 2019).

El giro de la muñeca se determina con la tabla 4.

Tabla 4. Puntuación giro muñeca

\begin{tabular}{ll}
\hline Posición & Puntuación \\
\hline Pronación o supinación media & $\mathbf{1}$ \\
Pronación o supinación extrema & $\mathbf{2}$ \\
\hline
\end{tabular}

Nota: Esta puntuación es independiente y no se añade puntuación modificada de la muñeca.

Fuente: (Cobo Sevilla, 2018).

Se sigue con la evaluación del Grupo B empezando por la puntuación del cuello ver tabla 5 .

Tabla 5. Puntuación cuello

\begin{tabular}{ll}
\hline Posición & Puntuación \\
\hline Flexión entre $0^{\circ}$ y $10^{\circ}$ & $\mathbf{1}$ \\
Flexión $>10^{\circ}$ y $\leq 20^{\circ}$ & $\mathbf{2}$ \\
Flexión $>20^{\circ}$ & $\mathbf{3}$ \\
Extensión en cualquier grado & $\mathbf{4}$ \\
\hline
\end{tabular}

Nota: La medición del cuello se obtiene del ángulo de flexión / extensión. Se suma 1 punto si la cabeza esta rotada o inclinada. Fuente: (Quinde Alvear Geovanny, 2017).

La posición del tronco se puntúa con la tabla 6.

Tabla 6. Puntuación tronco

\begin{tabular}{ll}
\hline Posición & Puntuación \\
\hline Sentado, bien apoyado y con un ángulo tronco-caderas & $\mathbf{1}$ \\
$>90^{\circ}$ & \\
Flexión entre $0^{\circ}$ y $20^{\circ}$ & $\mathbf{2}$ \\
Flexión $>20^{\circ}$ y $\leq 60^{\circ}$ & $\mathbf{3}$ \\
Flexión $>60^{\circ}$ & $\mathbf{4}$
\end{tabular}

Nota: La medición del tronco se obtiene del ángulo de flexión y dependerá si el trabajador está sentado o de pie. Se suma 1 punto si el tronco esta rotado o inclinado. Fuente: (Rodríguez Ruíz Yordán, 2015).

Luego de las penalizaciones sobre el tipo de actividad e instauración de fuerza se obtiene la puntuación final, ver tabla 7. 
Tabla 7. Niveles actuación según puntuación obtenida

\begin{tabular}{lll}
\hline Puntuación & Nivel & Actuación \\
\hline 1 o 2 & $\mathbf{1}$ & $\begin{array}{l}\text { Riesgo Aceptable } \\
\text { Pueden requerirse cambios en la tarea; es } \\
\text { o } 4\end{array}$ \\
& $\mathbf{2}$ & $\begin{array}{l}\text { conveniente profundizar en el estudio } \\
5 \text { o } 6\end{array}$ \\
7 & $\mathbf{3}$ & $\begin{array}{l}\text { Se requiere el rediseño de la tarea } \\
\text { Se requieren cambios urgentes en la tarea }\end{array}$ \\
\hline
\end{tabular}

Nota: Las medidas que se tomaran y el tiempo en el que se efectúen depende de la puntuación final. Fuente: (Cevallos, 2020)

\section{Significación de variables}

En esta etapa de la investigación se realizó un estudio de los elementos del método que son más significativos en el resultado final, y que se pueden modificar en condiciones reales. (Arana de las Casas Nancy Ivette, 2017). Este paso se realizó con la ayuda de una herramienta informática de software libre de la Universidad de Valencia disponible en su sitio web. Finalmente se recomendó la mejor de las opciones que determina una atenuación del nivel de riego por carga postural. (Cuixart, 2016).

\section{Resultados}

\section{Resultado del estudio de trabajo:}

En la tabla 8 se observa el resultado del estudio de trabajo para el puesto de cajera en un supermercado.

Tabla 8. Descripción del puesto

\begin{tabular}{ll}
\hline Nombre del puesto & Cajera \\
Descripción & Cobrar y despachar \\
Empresa & La Favorita \\
Departamento & Caja \\
Sección & 3 \\
Edad & 29 \\
Antigüedad en el puesto & 3 años \\
Tiempo que ocupa el cargo & 5 horas \\
Duración de su jornada laboral & 8 horas \\
Actividad principal & Pagar-cobrar \\
Parte comprometida $*$ & Extremidades superiores \\
Ciclos & $2-12$ minutos/cliente \\
Proceso macro & Ventas \\
Periodos de recuperación & 2 periodos de recuperación \\
Duración de su jornada laboral & 8 horas \\
\hline Nota: $*$ Partes del cuerpo que se comprometen con la ejecución de la tarea principal. Datos \\
referenciales del puesto del trabajador encargado de clocar la mercadería en los estantes. La \\
observación de campo se realizó por puesto no por grupo homogéneo. Fuente: (Cevallos, 2020)
\end{tabular}


En la tabla 9 se presenta los resultados finales al aplicar el método RULA, se consideró carga máxima de trabajo.

Tabla 9. Evaluación de postura por grupos RULA

\begin{tabular}{|c|c|c|c|}
\hline \multicolumn{4}{|c|}{ Grupo A (Extremidades superiores) } \\
\hline ITEM & Detalle de la posición & $\begin{array}{l}\text { Valor lado } \\
\text { derecho }\end{array}$ & $\begin{array}{l}\text { Valor } \\
\text { lado } \\
\text { izquierdo }\end{array}$ \\
\hline $\begin{array}{l}\text { Ángulo del } \\
\text { brazo }\end{array}$ & $\begin{array}{l}\text { El brazo esta entre } 21 \text { y } 45 \text { grados de } \\
\text { flexión o más de } 20 \text { grados de extensión. } \\
\text { Lado izquierdo se apoya lado derecho } \\
\text { rotado }\end{array}$ & 3 & 4 \\
\hline $\begin{array}{l}\text { Ángulo del } \\
\text { antebrazo }\end{array}$ & $\begin{array}{l}\text { El antebrazo esta entre } 60 \text { y } 100 \text { grados de } \\
\text { flexión. Lado derecho cruza línea central } \\
\text { del cuerpo }\end{array}$ & 2 & 3 \\
\hline $\begin{array}{l}\text { Ángulo de la } \\
\text { muñeca }\end{array}$ & $\begin{array}{l}\text { La muñeca está entre } 1 \text { y } 15 \text { grados de } \\
\text { flexión o extensión. Las dos muñecas } \\
\text { están desviadas radialmente }\end{array}$ & 3 & 3 \\
\hline $\begin{array}{l}\text { Ángulo de } \\
\text { giro de la } \\
\text { muñeca }\end{array}$ & $\begin{array}{l}\text { La muñeca está en posición de pronación } \\
\text { o supinación en rango medio }\end{array}$ & 1 & 1 \\
\hline \multicolumn{4}{|c|}{ Grupo B (Cuello, tronco y extremidades inferiores) } \\
\hline $\begin{array}{l}\text { Ángulo de } \\
\text { flexión del } \\
\text { cuello }\end{array}$ & $\begin{array}{l}\text { El cuello está flexionado entre } 10 \text { y } 20 \\
\text { grados. El cuello esta rotado }\end{array}$ & 3 & 3 \\
\hline $\begin{array}{l}\text { Ángulo de } \\
\text { flexión del } \\
\text { tronco }\end{array}$ & $\begin{array}{l}\text { El tronco está flexionado entre } 0 \text { y } 20 \\
\text { grados. Los dos lados demuestran torsión }\end{array}$ & 3 & 3 \\
\hline $\begin{array}{l}\text { Posición de } \\
\text { las piernas }\end{array}$ & $\begin{array}{l}\text { El trabajador está sentado con las piernas } \\
\text { y pies bien apoyados }\end{array}$ & 1 & 1 \\
\hline \multicolumn{4}{|c|}{ Fuerza (Actividad muscular y fuerza) } \\
\hline $\begin{array}{l}\text { Tipo de } \\
\text { actividad }\end{array}$ & $\begin{array}{l}\text { Actividad estática, se mantiene durante } \\
\text { más de un minuto seguido o es repetitiva }\end{array}$ & 1 & 1 \\
\hline $\begin{array}{l}\text { Fuerzas } \\
\text { ejercidas }\end{array}$ & $\begin{array}{l}\text { La carga o fuerza es menor de } 2 \mathrm{~kg} \text { y se } \\
\text { realiza intermitentemente }\end{array}$ & 0 & 0 \\
\hline
\end{tabular}

Nota: Los valores de la tabla se tomaron en condiciones máximas de trabajo en el mismo turno y horario. Fuente: (Cevallos, 2020)

En la tabla 10 se presenta el resultado del nivel de riesgo final con el método RULA: 
Tabla 10. Resultado final RULA

\begin{tabular}{lll}
\hline Puntuación (Rula) & Nivel & Actuación \\
\hline 6 & $\mathbf{4}$ & $\begin{array}{l}\text { Se requiere cambios rápidos en el diseño de } \\
\text { la tarea y/o del puesto de trabajo }\end{array}$ \\
\hline
\end{tabular}

Nota: Resultado de la ponderación 3 donde se analiza que se requiere cambios en el diseño de la tarea del trabajador antes que el daño sea mayor. Fuente: (Cevallos, 2020)

\section{Resultado de análisis de significación de variables}

En la tabla 11 se presenta el estudio de las variables administrativas más relevantes que inciden el valor final del nivel de riego con el método RULA:

Tabla 11. Resultado de la significación de las variables propias del puesto.

\begin{tabular}{lll}
\hline ITEM & SIGNIFICACIÓN & $\begin{array}{l}\text { Influencia en el nivel } \\
\text { de riesgo }\end{array}$ \\
\hline Edad & No incide & No \\
Antigüedad en el puesto & No incide & No \\
Tiempo que ocupa el cargo & No incide & No \\
Duración de su jornada laboral* & No incide & No \\
Actividad principal & No incide & No \\
\hline
\end{tabular}

Nota: * Se determino según datos de talento humano en el último periodo normal de trabajo Fuente: (Cevallos, 2020)

En la tabla 12 se presenta el estudio de las variables operativas más relevantes que inciden el valor final del nivel de riego con el método RULA:

Tabla 12. Resultado de la significación de las variables operativas del RULA.

\begin{tabular}{|c|c|c|}
\hline Variable & Importancia & $\begin{array}{l}\text { Influencia en el nivel de } \\
\text { riesgo }\end{array}$ \\
\hline Ángulo de flexión del brazo & No incide & $\mathrm{No}$ \\
\hline Posición del brazo & Medía & $\mathrm{Si}$ \\
\hline $\begin{array}{l}\text { Ángulo de flexión del } \\
\text { antebrazo }\end{array}$ & No incide & No \\
\hline $\begin{array}{l}\text { Ángulo de flexión de la } \\
\text { muñeca }\end{array}$ & No aplica & No \\
\hline Ángulo de giro de la muñeca & No aplica & No \\
\hline Ángulo de flexión del cuello & Medía & $\mathrm{Si}$ \\
\hline Ángulo de flexión del tronco & Medía & $\mathrm{Si}$ \\
\hline $\begin{array}{l}\text { Posición de las piernas del } \\
\text { trabajador }\end{array}$ & Medía & $\mathrm{Si}$ \\
\hline Tipo de actividad muscular & Alta & $\mathrm{Si}$ \\
\hline Fuerzas ejercidas & No aplica & No \\
\hline
\end{tabular}

Nota: Valores generados en las mismas condiciones de trabajo considerando carga máxima. 


\section{Diseño del puesto de trabajo}

En la figura 1. se presenta la modificación del plano de trabajo para mejorar 5 ángulos de incidencia: Posición del brazo, Ángulo de flexión del cuello, Tipo de actividad muscular, Ángulo de flexión del tronco con la reubicación de plano de trabajo, En la figura $1 \mathrm{Se}$ presenta la condición inicial y final de la propuesta de rotación de 90 grados del plano de trabajo para atenuar los valores altos según el método RULA.

Figura 1 Rediseño del puesto de trabajo de cajera

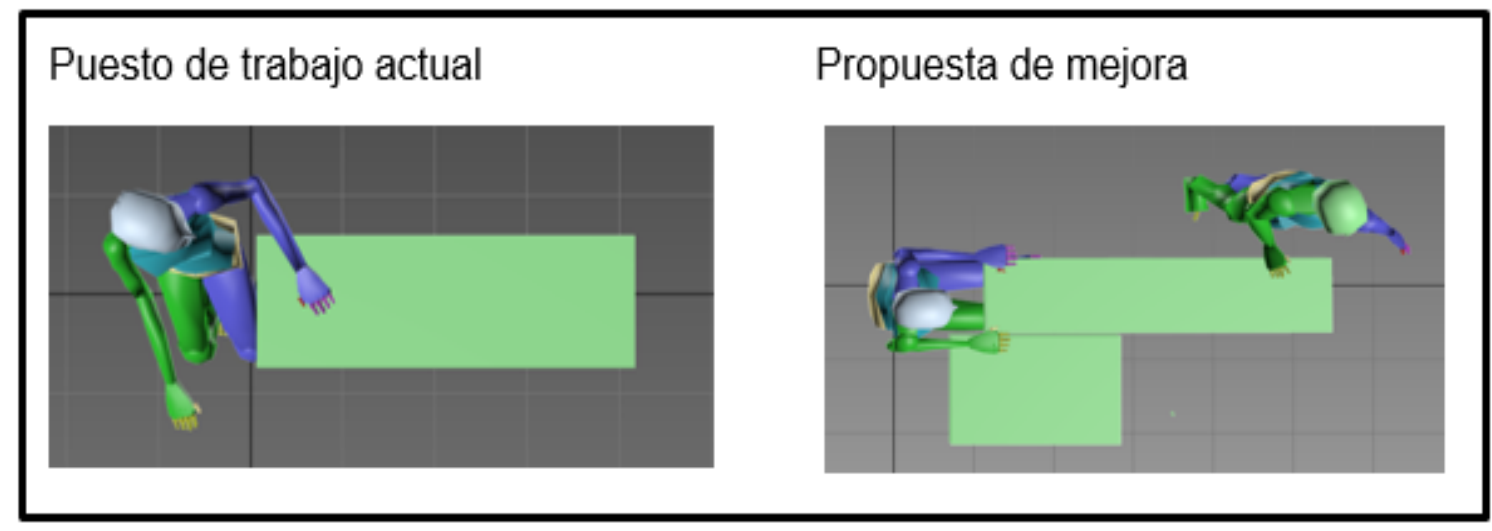

Fuente: (Cevallos, 2020)

En la tabla 13 se presenta la disminución del nivel de riesgo por RULA luego de la propuesta de diseño

Tabla 13. Resultado de aplicación del diseño

\begin{tabular}{|c|c|c|c|}
\hline Variable & Actividad & $\begin{array}{ll}\text { Nivel riesgo } \\
\text { inicial }\end{array}$ & $\begin{array}{l}\text { Nivel de riesgo } \\
\text { final }\end{array}$ \\
\hline Posición del brazo & $\begin{array}{l}\text { La carga no está } \\
\text { soportada sólo por el } \\
\text { brazo, sino que existe un } \\
\text { punto de apoyo }\end{array}$ & 6 & 2 \\
\hline $\begin{array}{l}\text { Ángulo de flexión del } \\
\text { cuello del trabajador }\end{array}$ & $\begin{array}{l}\text { El cuello está entre } 0 \text { y } 10 \\
\text { grados de flexión }\end{array}$ & 6 & 2 \\
\hline $\begin{array}{l}\text { Ángulo de flexión del } \\
\text { tronco del trabajador }\end{array}$ & $\begin{array}{l}\text { El tronco está flexionado } \\
\text { entre } 0 \text { y } 20 \text { grados }\end{array}$ & 6 & 2 \\
\hline $\begin{array}{l}\text { Posición de las piernas } \\
\text { del trabajador }\end{array}$ & $\begin{array}{l}\text { El trabajador está de pie } \\
\text { con el peso del cuerpo } \\
\text { distribuido en ambas } \\
\text { piernas y espacio para } \\
\text { cambiar de posición }\end{array}$ & 6 & 2 \\
\hline
\end{tabular}


Tipo de actividad Actividad dinámica, es 6

muscular del ocasional y no duradera

trabajador

Nota: Resultado en donde se toma en cuenta las variables que posiblemente se pueden mejorar para que la puntuación del diseño mejore. Adaptado de www.ergonautas.com

Los resultados demuestran una mejora significativa, pero en la realidad el diseño involucra cambio en todo el sistema de cobranza debiendo continuar este trabajo con un análisis de factibilidad financiera. Además de trabajos similares se anota que es fundamental la modificación de la silla para atenuar el nivel de riesgo ergonómico. (Paola Vernaza Pinzón, 2016).

\section{Conclusiones}

- El nivel de riesgo por posturas forzadas disminuye 4 puntos del resultado final RULA considerando: poner un punto de apoyo carga para que sea soportada, el cuello del trabajador debe estar entre $0^{\circ}$ y $10^{\circ}$ grados de flexión, el tronco debe estar flexionado entre $0^{\circ}$ y $20^{\circ}$, los brazos deben estar flexionados entre $20^{\circ}-45^{\circ}$; los antebrazos entre $60^{\circ}-100^{\circ}$; la muñeca debe ejercer pronación además el trabajador debe estar de pie con el peso del cuerpo distribuido en ambas piernas, y sobre el plano de trabajo debe girarse $90^{\circ}$ y la mesa para despacho en el lado derecho. Las características del puesto son para la región sierra y condiciones normales de presión y temperatura.

\section{Referencia bibliografía}

Arana de las Casas Nancy Ivette, S. Z. (2017). Evaluación ergonómica de la empresa Procesos y Servicios Industriales utilizando los métodos RULA, LEST y procesamiento de imágenes.

Avendaño Avendaño Sandra Bibiana, V. R. (2020). Análisis de factores de riesgo asociados a Desórdenes Músculo Esqueléticos en miembros superiores en trabajadores de la Dirección de Sanidad de la Policía Nacional.

Beatriz Capezzuto, M. G. (2017). Estrés laboral Síndrome de burnout y mobbing en el equipo de salud.

Campos, Y. C. (2017). Prevalencia de riesgos ergonómicos en una entidad lubricadora: aplicación del método rula y reba.

Cobo Sevilla, V. d. (2018). "Riesgos de desarrollar trastornos musculoesqueléticos en el personal de corte de calzado evaluado a través del método rula de forma manual". https://repositorio.uta.edu.ec/jspui/handle/123456789/28573

Cuellar Mendoza Jonathan, D. S. (2018). Método RULA.

Cuixart, S. N. (2016). Evaluación de las condiciones de trabajo: carga postural. 
Flores, M. G. (2018). Evaluación de riesgos ergonómicos en el área de estibación y monitoreo de panel central, mediante los métodos rula y ocra, en industrias guapán.

Gómez, C. A. (2017). La investigación científica y las formas de titulación. Aspectos conceptuales y prácticos. Quito: Jurídicamdel Ecuador.

Henao Robledo, F. (2016). Diagnóstico integral de las condiciones de trabajo y salud. Bogota: $2^{\circ}$ editorial.

Jara, O. V. (2018). Diseño del puesto de trabajo en dependientes de farmacia de una cadena farmacéutica de ecuador.

Jessica Jazmin Pesina Lumbreras, E. G. (2019). Comparación de técnicas actuales y futuras de evaluación ergonómica.

Kolodziej, S. F. (2019). Análisis de un puesto de trabajo en el sector de obras pública de la municipalidad de oberá, misiones.

Levano Boggiano, V. A. (2018). Prevalencia y características del dolor cervical en cajeras de supermercados de Lima Metropolitana.

Moreno Fiallos Victor, Z. M. (2019). Inserción laboral de personas con discapacidad física y diseño de un modelo de puesto de trabajo ergonómico: centros laborales del sector público.

Moreno Medina, V. H. (2018). Análisis de métodos específicos en factores de riesgo ergonómico de puesto de trabajo: manipulación manual de cargas, movimientos repetitivos, posturas forzadas de trabajo, en el personal de área de mantenimiento de equipo pesado en el gobierno de pastaza.

Moya Reyes, M. A. (2017). Evaluación del riesgo ergonómico en lineas de ensacado mediante el método rula.

Ortiz, J. B. (2018). Relación entre posturas ergonómicas inadecuadas y la aparición de trastornos musculo esqueléticos en los trabajadores de las áreas administrativas que utilizan pantalla de visualización de datos.

Pablo E. Aliaga, J. I. (2016). La charla motivacional: Una estrategia para abordar el desconocimiento de factores de riesgo ergonómico en un supermercado chileno.

Paola Vernaza Pinzón, C. H. (2016). Dolor músculo-esquelético y su asociación con factores de riesgo ergonómicos, en trabajadores administrativos.

Quinde Alvear Geovanny, G. F. (2017). Evaluación de riesgos ergonómicos, en el área de estibación, monitoreo de panel central, enfermera, laboratorista y soldador, mediante los métodos rula y ocra, en industrias Guapán.

Quintana Salgado LA, M. S. (2020). Síntomas musculoesqueléticos asociados a posturas ergonómicas inadecuadas.

Rodríguez Ruíz Yordán, G. V. (2015). Empleo de los métodos erin y rula en la evaluación ergonómica de estaciones.

Saúl Rangel Lara, D. D. (2019). Evaluación ergonómica en trabajo de jardinería con el método RULA.

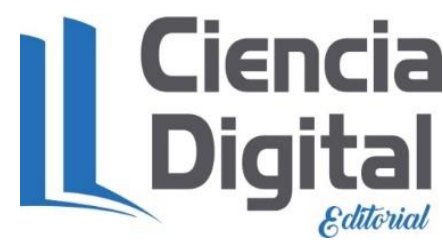




\section{PARA CITAR EL ARTÍCULO INDEXADO.}

Cevallos Tapia, L. A., Córdova Suárez, M. A., Vega Falcón, V., \& Villacres Cevallos, E. P. (2021). Diseño ergonómico del puesto de trabajo de cajera en supermercado con exposición a posturas incómodas. ConcienciaDigital, 4(2), 198-226. https://doi.org/10.33262/concienciadigital.v4i2.1662

\section{Ciencia \\ LDigital}

El artículo que se publica es de exclusiva responsabilidad de los autores y no necesariamente reflejan el pensamiento de la Revista Conciencia Digital.

El artículo queda en propiedad de la revista y, por tanto, su publicación parcial y/o total en otro medio tiene que ser autorizado por el director de la Revista Conciencia Digital.

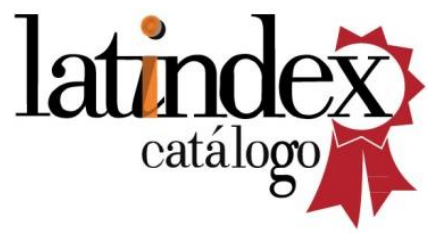

